\title{
Synthesis of porous yttria-stabilized zirconia microspheres by ultrasonic spray pyrolysis
}

\author{
Maria Perez-Page ${ }^{1}$, Ryan Guzalowski ${ }^{1}$, Dereck N. F. Muche ${ }^{2}$, Ricardo H. R. Castro ${ }^{2}$, Pieter
}

Stroeve ${ }^{1}$

\begin{abstract}
High yield synthesis of yttria-stabilized zirconia (YSZ) porous microspheres can enable application in advanced thermal barrier coatings, exploiting reflective properties originated from high temperature photonics. The complexity of typical wet-chemistry processes utilized in microspheres manufacturing limits yield and can hardly deliver stable porous structures. Here, ultrasonic spray pyrolysis was utilized to produce high quantities of porous YSZ. Droplets of an aqueous precursor solution were dragged with continuous air flow into a furnace kept at temperatures varying from 700 to $1200{ }^{\circ} \mathrm{C}$. Spherical fully cubic zirconia particles were obtained in all processing conditions, and the sizes of the spheres decreased as a function of the processing temperature, ranging from 484 to $373 \mathrm{~nm}$ (average diameters), while their crystallite sizes had an inverse trend, ranging from 4.9 to $25.2 \mathrm{~nm}$. This was attributed to higher temperatures activating coarsening of the spheres.
\end{abstract}

Keywords: Processing, ceramics, synthesis, microspheres 


\section{Introduction}

Zirconia microspheres have been recently proposed as base materials for thermal barrier coatings (TBCs) as they combine low thermal conductivity and an associated reflection of thermal radiation [1-3]. This reflection results from the resonant behavior of the spheres that creates discrete light states where every sphere acts as a meta-atom for light [3]. Control of sphere sizes is critical for this application since the radiation reflection has to be coherent with the diameter of the spheres. Sol-gel chemistry is currently the most reliable method to achieve refined control of the sizes, although the process yield is very limited, prohibiting scaling-up [4].

Spray pyrolysis is a continuous flow process widely used in industry to produce particles with a diameter larger than $5 \mu \mathrm{m}$ because of its versatility and relatively low producing cost [58]. The process is based on the thermal decomposition of aerosols (liquid micro-sized droplets). For the generation of oxide particles, aerosols are formed from a liquid solution containing ionic precursor (typically salts), and can be done via two-fluid atomization, liquid atomization by airspray, or ultrasonic atomization [9]. The aerosol is then fluxed into a heating zone using a carrier gas where the solvent evaporates, rapidly leading to a supersaturation condition inside the liquid droplets that causes precipitation of hydroxide phases. Such hydroxides are highly homogeneous in terms of the ionic distribution since those are mixed in the liquid phase and quickly precipitated. The hydroxides are then transformed into oxides if enough temperature or residence time (total time the particles flow inside the heating zone) is provided in the heating zone $[5,6]$.

In the present study, an ultrasonic atomization device was used to produce hollow yttriastabilized cubic zirconia (YSZ) microspheres for the first time. In contrast to previous literature $[8,10]$, powder analyses showed that the spheres had a core-shell structure, with a hard exterior and porous interior. Such hollow structures have not been reported previously but are 
theoretically highly suitable for TBCs due to improved heat insulation capabilities. The temperature of the heating zone was varied from 700 to $1200{ }^{\circ} \mathrm{C}$ during the Ultrasonic Spray Pyrolysis, UPS, while other parameters were kept constant. Spherical particles were obtained with retention of small crystallite sizes.

\section{Experimental Procedures}

Synthesis by Ultrasonic Spray Pyrolysis

A schematic diagram of the USP reactor used in this study is shown in Fig. 1. The major components of the system are: (1) ultrasonic spray chamber with an ultrasonic nebulizer working at $1.7 \mathrm{MHz}$, (2) solution flask supplying the precursor solution into the chamber, (3) air as a carrier gas, (4) alumina tube furnace (GSL-1700X, MTI corporation) as the reaction chamber, (5) precipitator chamber composed of an electrostatic precipitator working at $1.5 \mathrm{kV}$ and a silicon heat tape to avoid condensation of water prior the powder collection, (6) DC voltage supply to power the precipitator, and (7) bubbler with water to capture by-products from the reaction and allow vapors to escape.

Zirconium (IV) oxynitrate hydrate 99\% (Sigma Aldrich) and yttrium (III) nitrate hexahydrate $99.9 \%$ (Alfa Aesar) were used as ionic precursors. The solution was prepared to obtain $10 \%$ mol of $\mathrm{Y}_{2} \mathrm{O}_{3}$ to $90 \mathrm{~mol} \% \mathrm{ZrO}_{2}$ by dissolving zirconium oxynitrate and yttrrium nitrate hexahydrate to form $0.2 \mathrm{M}$ in deionized water. The reaction was performed at 700,800 , $900,1000,1100$ and $1200{ }^{\circ} \mathrm{C}$, all temperatures measured at the center of the alumina tube with internal diameter $72 \mathrm{~mm}$, external diameter $80 \mathrm{~mm}$ and $1000 \mathrm{~mm}$ in length. The edges of the tube were kept at $\sim 400{ }^{\circ} \mathrm{C}$. Air is used as carrier gas; the flow rate was $8 \mathrm{~L} / \mathrm{min}$. To collect the synthesized particles from the electrostatic precipitator, the device was rinsed with ethanol after 
termination of the USP reaction. The ethanol mixture, containing the synthesized particles, was put into centrifuge tubes and centrifuged for $5 \mathrm{~min}$ at $3400 \mathrm{rpm}$. The supernatant, containing mainly ethanol, was then discarded. The tubes were dried in an oven at $100{ }^{\circ} \mathrm{C}$ for at least $12 \mathrm{~h}$.

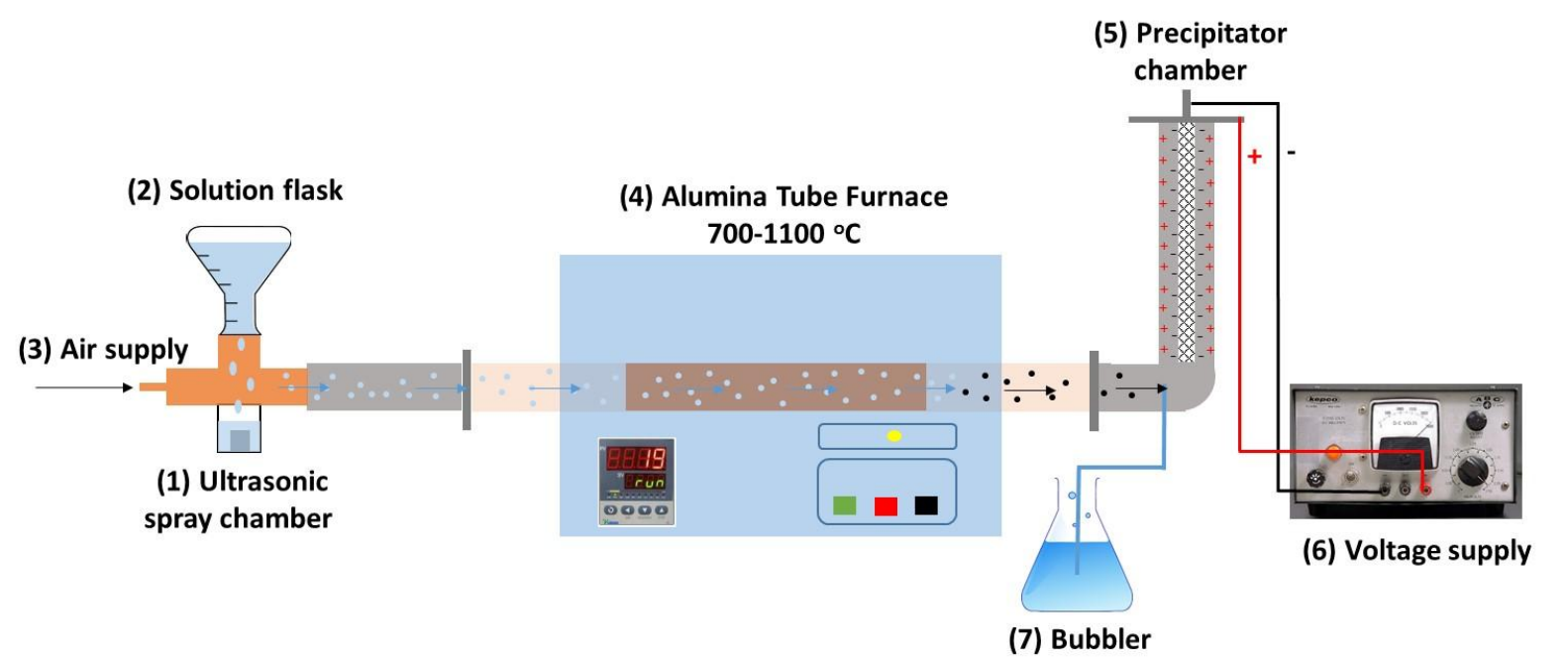

Figure 1: Schematic diagram of Ultrasonic Spray Pyrolysis reactor.

\section{Characterization Techniques}

A Bruker-AXS D8 Advancer diffractometer (Bruker-AXS, Inc) operating at $40 \mathrm{kV}$ and 40 $\mathrm{mA}$ with $\mathrm{CuK} \alpha$ radiation $(\lambda=0.15406 \mathrm{~nm})$ was used to collect $\mathrm{X}$-ray diffraction powder patterns from all samples. The data were collected over a range of $20-90^{\circ}(2 \theta)$ with a step size of $0.032^{\circ}$. Samples were mounted on zero-background holders. Crystallite sizes were obtained from diffraction peak broadening (Scherrer's method), utilizing a whole profile fitting implemented with the JADE 6.1 (MDI) software. $\mathrm{LaB}_{2}$ was mixed as standard to account for instrumental functions.

Samples were analyzed using Scanning Electron Microscope equipped with Energy Dispersive X-ray Spectroscopy (SEM/EDS) to determine particle size, size distribution, and composition. The powders were lightly grounded and dusted onto double-side carbon tape and 
sputter-coated with gold for two minutes to improve the conductivity using a plasma sputtering coater (Denton Vacuum Desk II). Images were taken with a Philips XL30 SFEG SEM at $5 \mathrm{~kW}$ with a TLD detector at different magnifications, with EDS taken at $5 \mathrm{~kW}$. Composition analyses revealed all samples had consistently $10 \mathrm{~mol} \%$ Yttria (see Supplemental Materials, Table S1). For particle size distribution, at least 100 particles were measured. A JEOL 25000 Transmission Electron Microscopy was also used to image the YSZ particles. TEM was operated at $200 \mathrm{keV}$ with samples dispersed in ethanol and dripped onto a carbon coated cooper grid.

\section{Results and Discussion}

Figure 2 shows XRD patterns of YSZ particles produced by USP at different temperatures, as indicated. The diffraction peaks become more intense and narrow with the increase of the temperature of synthesis, which implies an increase of the crystallite size listed in the figure.

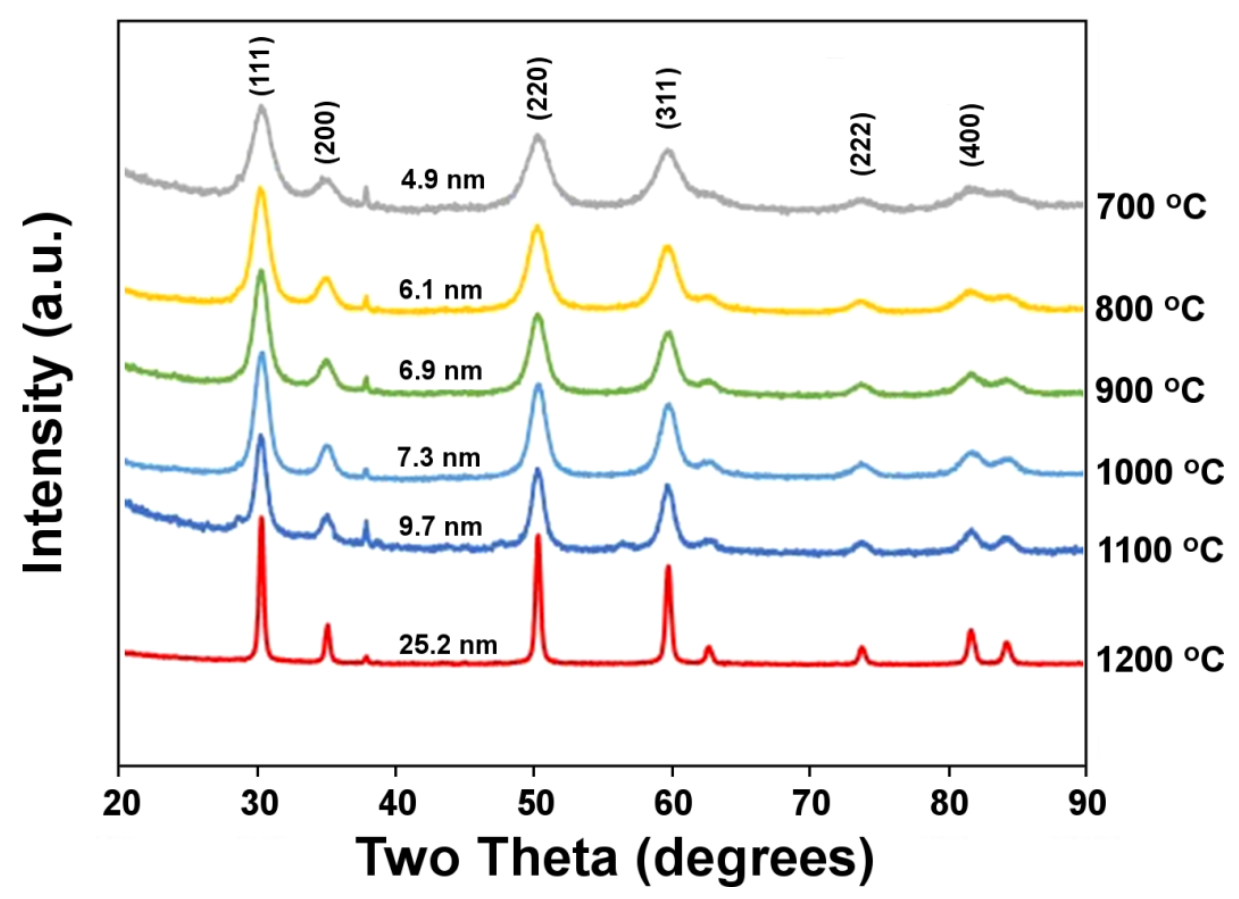

Figure 2: XRD patterns of YSZ particles obtained from USP at indicated temperatures. 
Table S1 shows the lattice parameters for all samples, averaging $5.1446 \AA$ with small nonsystematic deviations $(0.001 \AA)$ as a function of temperature. These numbers are all consistent with reports for zirconia powders with similar Y content [11]. Figure 3 shows SEM images of the produced particles and the corresponding particle size distribution for the samples synthesized at 700,1100 and $1200{ }^{\circ} \mathrm{C}$ (fata for other temperatures omitted for brevity). The images reveal isotropic spheres with a relatively broad size distribution, with the average diameter slightly decreasing with increasing temperature. Moreover, one observes that the surface roughness increases with increasing temperature, such that for the samples prepared at $1200{ }^{\circ} \mathrm{C}$, highly granulated spherical particles are observed.

The observations can be attributed to the dynamics of the particle formation [7]; i.e. as the round liquid droplets containing the ionic precursors enter the reactive zone, five main processes take place: isotropic evaporation of the solvent, precipitation of hydroxide phases as supersaturation of the solution is met, complete drying of solvent, followed by decomposition of the hydroxides into oxides, and sintering of the oxide phases. At relatively low temperatures, evaporation of the solvent and precipitation of the hydroxides are expected to occur concomitantly, followed by decomposition, but due the higher activation energies, sintering is hindered. This results in relatively large spheres with small crystallite sizes (see Figure 1), microscopically observed as smooth surfaces in the SEM images in Figure 3 [12]. At higher temperatures, sintering is also activated, leading to more shrinkage of the spheres and crystallite growth, which is consistent with the much rougher surface of the particles shown in Figure 3 for the samples produced at $1200{ }^{\circ} \mathrm{C}$. 

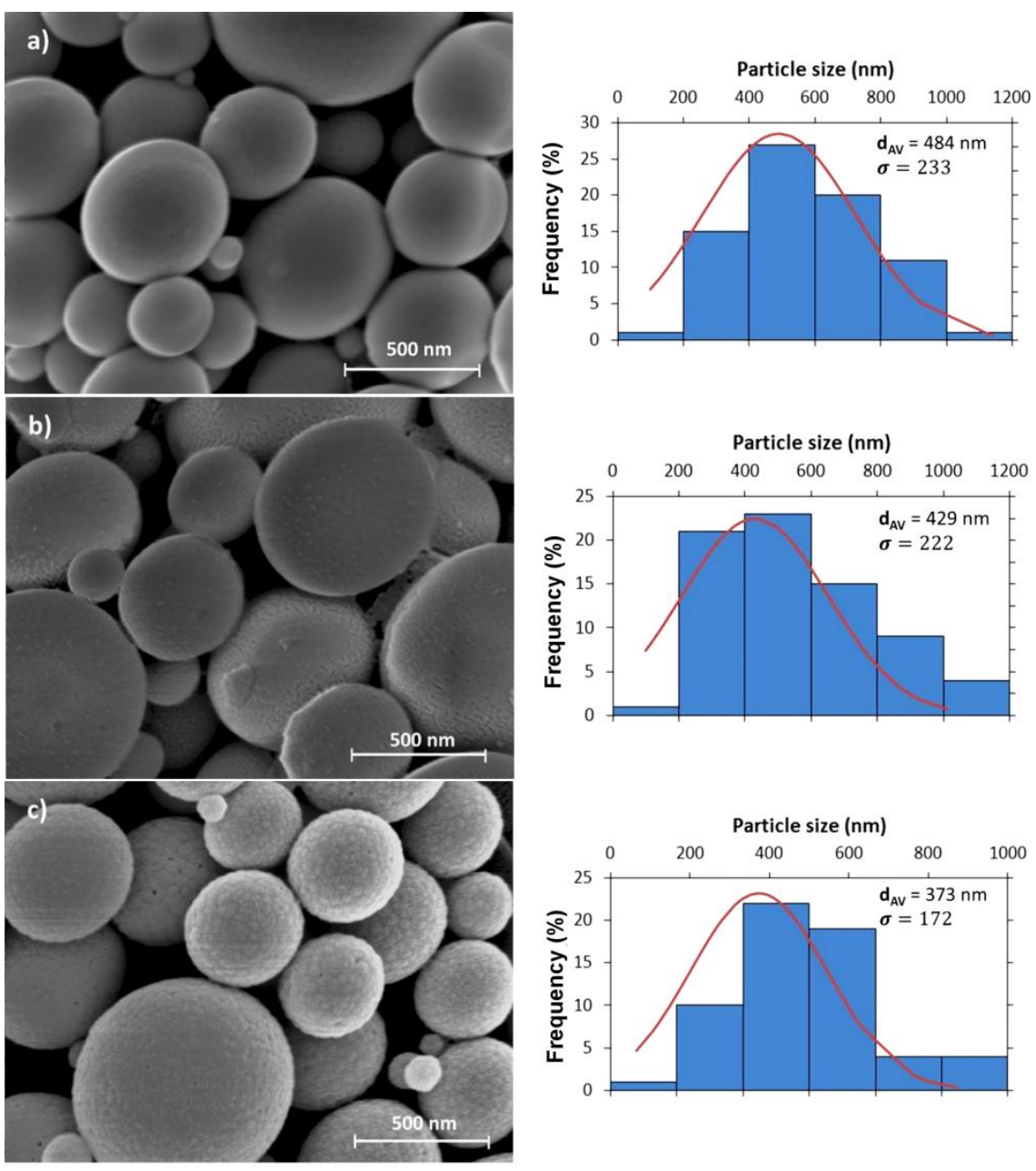

Figure 3. SEM images of spheres produced at (a) 700, (b) 1100 and (c) $1200{ }^{\circ} \mathrm{C}$ and respective size distribution.

Figure 4 shows TEM images corresponding to YSZ synthesized at 700 and $1100{ }^{\circ} \mathrm{C}$. Consistently with SEM, the samples produced at $1100{ }^{\circ} \mathrm{C}$ show stronger contrast, suggesting denser aggregates forming the sphere as compared to the sample prepared at $700{ }^{\circ} \mathrm{C}$. However, both samples did not present a highly dense interior [10] (which would not have allowed electron transmittance). This suggests that the heating rates of the liquid droplets were fast enough to cause precipitation from the edges of the droplets, creating a precipitation front moving inwards. Because of the limited supply of ions, it results in a relatively porous interior and a hard shell. In 
practice, this heating rate can be controlled by the carrier gas velocity, but a systematic work relating these parameters is beyond the scope of this work. This condition has been theoretically predicted but not demonstrated [7], and is fundamentally distinct from the growth of other spherical particles of fluorite structures prepared by wet-chemistry routes, such as $\mathrm{CeO}_{2}[13,14]$, in which dense spheres with small crystallite sizes are formed and incur coarsening at high temperatures so that small grains are consumed by larger ones to ultimately form a solid particle composed by a single crystallite.
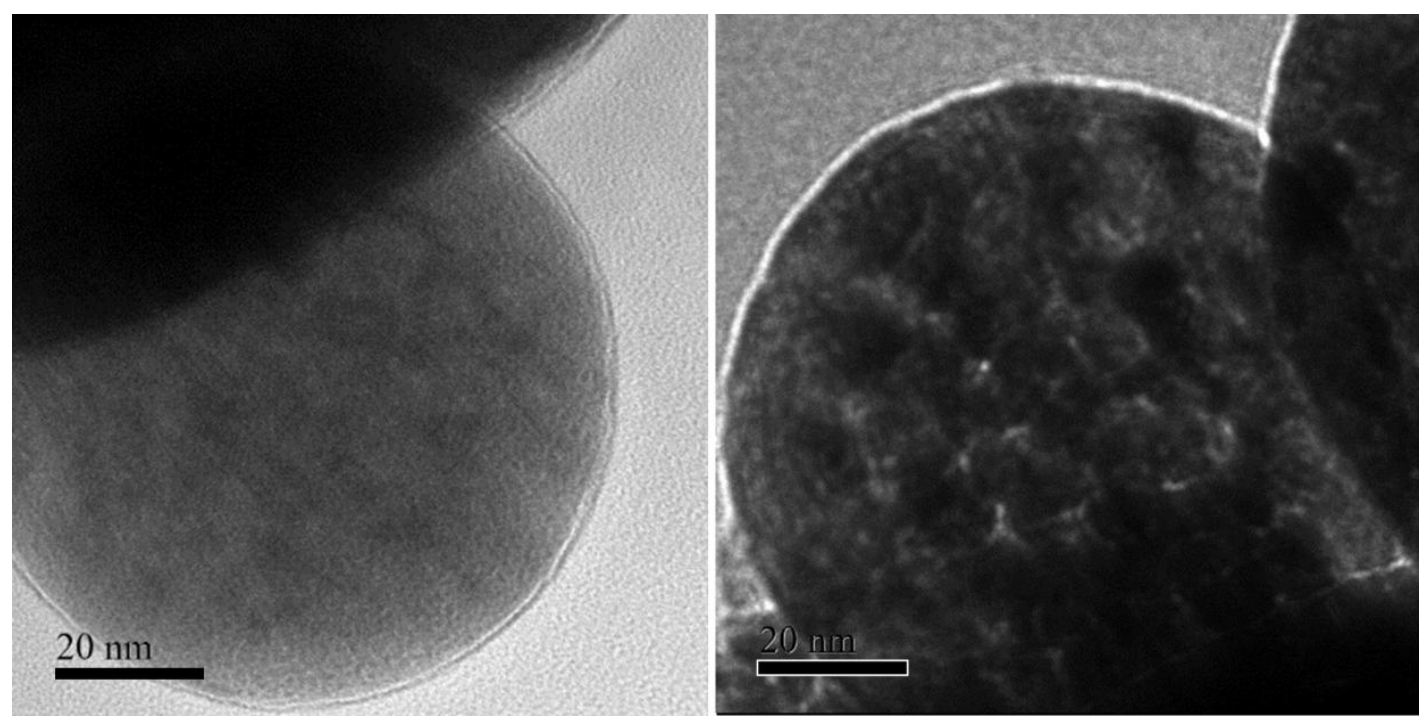

Figure 4. TEM images of YSZ spheres synthesized at 700 (left) and $1100{ }^{\circ} \mathrm{C}$ (right).

\section{Acknowledgements}

This work was supported by the Department of Energy [National Nuclear Security Administration] under Award Number DE-NE0000704. 


\section{References}

[1] D. R. Clarke, C. G. Levi. Ann. Rev. Mater. Res. 33 (2003) 383.

[2] V. Shklover, L. Braginsky, G. Witz, M. Mishrikey, C. Hafner. J. Comput. Theor. Nanosci. 5 (2008) 862.

[3] P. N. Dyachenko, J. J. do Rosario, E. W. Leib, A. Y. Petrov, R. Kubrin, G. A. Schneider, H. Weller, T. Vossmeyer, M. Eich. ACS Photonics 1 (2014) 1127.

[4] E. W. Leib, R. M. Pasquarelli, M. Blankenburg, M. Müller, A. Schreyer, R. Janssen, H. Weller, T. Vossmeyer. Part. Part. Syst. Char. 33 (2016) 645.

[5] S. C. Tsai, Y. L. Song, C. S. Tsai, C. C. Yang, W. Y. Chiu, H. M. Lin. J. Mater. Sci. 39 (2004) 3647.

[6] J. H. Bang, K. S. Suslick. Adv. Mater. 22 (2010) 1039.

[7] G. L. Messing, S.-C. Zhang, G. V. Jayanthi. J. Am. Ceram. Soc. 76 (1993) 2707.

[8] E. Djurado, E. Meunier. J. Solid State Chem. 141 (1998) 191.

[9] M. T. T. Camargo, Q. Jacques, L. B. Caliman, J. Miagava, D. Hotza, R. H. R. Castro, D. Gouvea. Mater. Lett. 171 (2016) 232.

[10] B. Dubois, D. Ruffier, P. Odier. J. Am. Ceram. Soc. 72 (1989) 713.

[11] J.A. Krogstad, M. Lepple, Y. Gao, D.M. Lipkin, C.G. Levi CG. J Am Ceram Soc. 94 (2011) 4548.

[12] M. K. Sharma, P. Rohani, S. Liu, M. Kaus, M. T. Swihart. Langmuir 31 (2015) 413.

[13] G.I. Nkou Boualaa, N. Claviera, J. Léchelleb, A. Mesbaha, N. Dacheuxa, R. Podora. Ceram. Int. $41(2015) 14703$.

[14] G. I. Nkou Bouala, N. Clavier, R. Podor, J. Cambedouzou, A. Mesbah, H. P. Brau, J. Léchelle, N. Dacheux. Cryst. Eng. Comm. 16 (2014) 6944. 